\title{
Electron-vibration relaxation in oxygen plasmas
}

\author{
V. Laporta, ${ }^{1,2, *}$ K. L. Heritier, ${ }^{3, \dagger}$ and M. Panesi ${ }^{3, \ddagger}$ \\ ${ }^{1}$ Istituto di Nanotecnologia, CNR, 70126 Bari, Italy \\ ${ }^{2}$ Department of Physics and Astronomy, \\ University College London, London WC1E 6BT, UK \\ ${ }^{3}$ University of Illinois at Urbana-Champaign, Urbana, Illinois, 61801
}

\begin{abstract}
An ideal chemical reactor model is used to study the vibrational relaxation of oxygen molecules in their ground electronic state, $\mathrm{X}^{3} \Sigma_{g}^{-}$, in presence of free electrons. The model accounts for vibrational non-equilibrium between the translational energy mode of the gas and the vibrational energy mode of individual molecules. The vibrational levels of the molecules are treated as separate species, allowing for non-Boltzmann distributions of their population. The electron and vibrational temperatures are varied in the range [0 - 20000] K. Numerical results show a fast energy transfer between oxygen molecules and free electron, which causes strong deviation of the vibrational distribution function from Boltzmann distribution, both in heating and cooling conditions. Comparison with Landau-Teller model is considered showing a good agreement for electron temperature range [2000 - 12000] K. Finally analytical fit of the vibrational relaxation time is given.
\end{abstract}

\section{INTRODUCTION}

The study of non-equilibrium relaxation in weakly ionized molecular plasmas is of great interest to the scientific community. Examples of applications include: atmospheric entry [1-3], optical diagnostics, discharges [4], plasma assisted combustion [5], material processing, just to name a few. This work is concerned with the study of non-equilibrium phenomena in high speed plasma flows relevant to the entry into Earth's atmosphere, in particular the electron-oxygen energy transfer will be considered, which study, in our opinion, has been largely overlooked by the aerothermodynamic community. Previous works on electron cooling by oxygen molecules in upper atmosphere can be found in the Ref. [6-9] and more recently by Jones et al. [10]

This research is at the interface between computational chemistry and computational fluid dynamics and aims at the development of reduced models based on microscopic theory and applying them to the macroscopic scale. Previously published research efforts were focused on the characterization of the $\mathrm{N}_{2}\left(\mathrm{X}^{1} \Sigma_{\mathrm{g}}^{+}\right)$kinetics, resulting from the interaction with $\mathrm{N}\left({ }^{4} \mathrm{~S}^{\mathrm{o}}\right)$ atoms [11-13] and free electrons [14-16]. In this paper, by using a state-to-state (STS) kinetic approach, we concentrate on the study of the energy exchange between electrons and vibrational energy for $\mathrm{O}_{2}\left(\mathrm{X}^{3} \Sigma_{g}^{-}\right)$molecules. In particular, we are interested in the study of non-equilibrium regimes characterized by low free electron temperature $(<10000 \mathrm{~K})$. In hypersonic applications, in fact, the electrons formed by collision impact between heavy

*Electronic address: vincenzo.laporta@nanotec.cnr.it

${ }^{\dagger}$ Electronic address: kheritie@illinois.edu

${ }^{\ddagger}$ Electronic address: mpanesi@illinois.edu 
particles are relatively cold, and the average energy of the electrons is bounded by the heavy particle energy.

Due to the lack of a dipole moment, low-energy electron-impact cross sections for direct excitation of vibrational states of homo-nuclear molecules, like oxygen or nitrogen, are, in general, negligible. However, the vibrational excitation in the case of $\mathrm{N}_{2}$ and $\mathrm{O}_{2}$ molecules can also proceed via the formation of an intermediate negative ion complex, the so-called resonant processes. A well known example is the $\mathrm{N}_{2}^{-}$resonance for nitrogen molecule. The study of $\mathrm{N}_{2}\left(\mathrm{X}^{1} \Sigma_{\mathrm{g}}^{+}\right)$excitation by electron-impact has been the object of considerable investigation both from the theoretical [14, 17-23] and experimental [24-26] point of view. For oxygen molecules, the minimum of the potential energy curve of $\mathrm{O}_{2}^{-}$lies below that of $\mathrm{O}_{2}$ and the position of the resonance is therefore very close to the cross section threshold [2729]. As result, the electron-vibration energy transfer in oxygen could be an efficient process at low temperature even if the magnitude of corresponding cross sections are not as large as those for nitrogen.

This work addresses the study of the vibrational energy transfer processes in an ideal chemical reactor filled with molecular oxygen and free electrons. Dissociation is neglected. In this reactor, cold oxygen molecules at room temperature, seeded with small amount of free electrons, are suddenly heated by several thousand degrees Kelvin driving the gas toward a strong non-equilibrium condition. The present investigation is devoted to the in-depth study of the relaxation of oxygen $\mathrm{O}_{2}\left(\mathrm{X}^{3} \Sigma_{g}^{-}\right)$molecules in presence of free electrons. Our proposed model relies on state-specific reaction rate coefficients of an ab-initio database developed as part of the Phys4Entry database [30]. The published results will serve as a benchmark for validation of more approximate theories reduced order models. Furthermore, the insights gained by the analysis of oxygen excitation will serve as guidelines for the development of reduced models. As first step in this direction we have extracted macroscopic relaxation rate parameters for the vibrational energy transfer of oxygen molecules in an electron gas. The derived parameters can be readily used in fluid-dynamic codes for the modeling nonequilibrium plasma flows.

The manuscript is organized as follow: Section II contains the physical model and the computational method used; In section III A the effect of the multi-quantum transitions is studied and in Section III B the results in the relaxation time formalism are presented. Conclusions are drawn in Section IV.

\section{PHYSICAL MODEL}

We wish to investigate the vibrational excitation of oxygen molecules, in their electronic ground state $\mathrm{X}^{3} \Sigma_{g}^{-}$, when subjected to sudden heating/cooling in an ideal chemical reactor, filled with hot/cold electrons. We make the following assumptions: (i) The mixture electron$\mathrm{O}_{2}$ is supposed to be kept in a isothermal bath at constant free-electron temperature $T_{e}$; (ii) Electrons are considered in equilibrium according to a Maxwell-Boltzmann distribution at temperature $T_{e}$, with a corresponding pressure given by,

$$
p_{e}=n_{e} k_{B} T_{e},
$$

where $n_{e}$ is the electron number of particles per unit volume and $k_{B}$ the Boltzmann constant; (iii) At the beginning of the numerical experiment, the population of the vibrational energy levels of the oxygen molecules is assumed to follow a Maxwell-Boltzmann distribution at 


\begin{tabular}{cccccc}
\hline \hline$v$ & $\epsilon_{v}$ & $v$ & $\epsilon_{v}$ & $v$ & $\epsilon_{v}$ \\
\hline 0 & 0.000 & 14 & 2.435 & 28 & 4.280 \\
1 & 0.196 & 15 & 2.587 & 29 & 4.382 \\
2 & 0.388 & 16 & 2.735 & 30 & 4.476 \\
3 & 0.573 & 17 & 2.881 & 31 & 4.565 \\
4 & 0.756 & 18 & 3.024 & 32 & 4.651 \\
5 & 0.937 & 19 & 3.164 & 33 & 4.730 \\
6 & 1.117 & 20 & 3.301 & 34 & 4.794 \\
7 & 1.291 & 21 & 3.436 & 35 & 4.847 \\
8 & 1.461 & 22 & 3.568 & 36 & 4.898 \\
9 & 1.629 & 23 & 3.696 & 37 & 4.938 \\
10 & 1.796 & 24 & 3.821 & 38 & 4.960 \\
11 & 1.960 & 25 & 3.942 & 39 & 4.976 \\
12 & 2.122 & 26 & 4.059 & 40 & 4.987 \\
13 & 2.281 & 27 & 4.172 & 41 & 4.994 \\
\hline \hline
\end{tabular}

TABLE I: Calculated vibrational levels of $\mathrm{O}_{2}\left(\mathrm{X}^{3} \Sigma_{g}^{-}\right)$molecule for rotational level $j=1$. Energies are given in $\mathrm{eV}$.

the internal temperature $T_{v}^{0} \neq T_{e}$; (iv) The volume of the chemical reactor is kept constant during the experiment and the thermodynamic system is closed (no mass exchange with surrounding environment); (v) The electronic excitation and ionization processes are not considered. The kinetic processes included in the numerical simulations are the vibrationalexcitations (VE) by electron-impact, i.e.:

$$
e+\mathrm{O}_{2}\left(\mathrm{X}^{3} \Sigma_{g}^{-} ; v, j\right) \rightleftarrows e+\mathrm{O}_{2}\left(\mathrm{X}^{3} \Sigma_{g}^{-} ; w, j\right),
$$

where $v, w \in \mathcal{V}=\{0, \ldots, 41\}$ are vibrational levels supported by the potential energy curve of electronic ground state of $\mathrm{O}_{2}$ for $j=1$ rotational level. The list of the vibrational energy levels $\epsilon_{v}$ used in this work is given in Table I.

The vibration relaxation process of the molecules in a chemical reactor can be described by means of a kinetic or STS approach. The vibrational distribution function (vdf) of the oxygen molecules, $n_{v}(t)$, in a particular energy level $v$ is then obtained by solving a system of master equations (ME),

$$
\frac{d n_{v}}{d t}=n_{e} \sum_{w \in \mathcal{V}}\left[k_{w, v} n_{w}-k_{v, w} n_{v}\right], \quad v \in \mathcal{V},
$$

where the electron impact rate coefficients $k_{v, w}\left(T_{e}\right)$ have been calculated from cross sections reported in Refs. [27, 28] assuming a Maxwell-Boltzmann distribution for free electrons. For electron energies of interest to this study, the excitation of the oxygen molecules, Eq. (2), proceeds via the formation of negative ion $\mathrm{O}_{2}^{-}$, with subsequent de-excitation. In calculating the cross sections, the ${ }^{2} \Pi_{g},{ }^{2} \Pi_{u},{ }^{4} \Sigma_{u}^{-},{ }^{2} \Sigma_{u}^{-}$resonant states have been included. Some results for $k_{v, w}$ are shown in Fig. 1 for mono- and multi-quantum vibrational transitions.

A different approach to modeling vibrational energy relaxation is due to Landau and Teller (LT) [31] and it is based on a number of assumptions that are in general valid for simple harmonic oscillator molecules. Within the LT formalism, the relaxation parameter, 

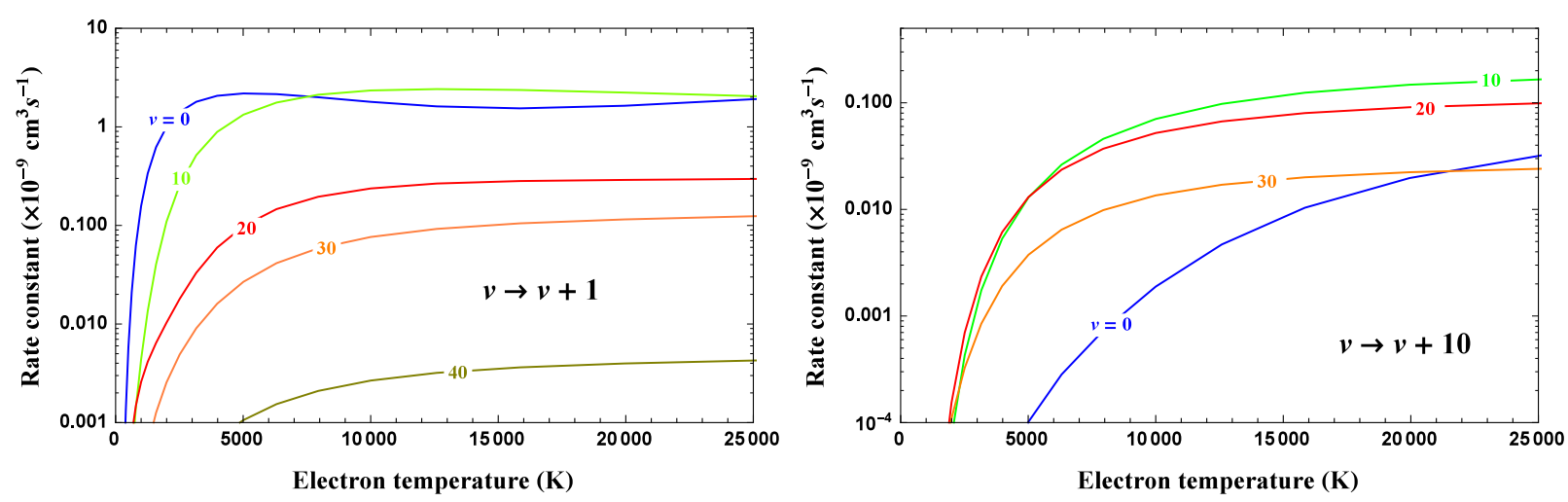

FIG. 1: Electron- $\mathrm{O}_{2}$ vibrational excitation rate constants for mono- $(w-v=1$, plot on the left) and multi-quantum $(w-v=10$, plot on the right) transitions for $j=1$. The results are taken from Refs. $[27,28]$

$\tau_{e}$, is defined as the characteristic time of a first order dynamical system response. The time evolution of oxygen vibrational energy $E_{v i b}$, given by:

$$
E_{v i b}(t)=\sum_{v \in \mathcal{V}} n_{v}(t) \epsilon_{v}
$$

is governed by the solution of first-order differential equation:

$$
\frac{d E_{v i b}}{d t}=\frac{E_{v i b}^{*}-E_{v i b}}{\tau_{e}}
$$

where $E_{v i b}^{*}$ is the equilibrium vibrational energy, at temperature $T_{e}$, defined by

$$
E_{v i b}^{*}=\sum_{v \in \mathcal{V}} n_{v}^{*}\left(T_{e}\right) \epsilon_{v}
$$

where $n_{v}^{*}\left(T_{e}\right)$ is the equilibrium $\mathrm{O}_{2}$ vdf.

\section{ELECTRON-VIBRATIONAL ENERGY TRANSFER}

In this section, the STS model, presented above, will be used to study the relaxation kinetics of the vibrationally excited states in the isothermal and isochoric reactor for a wide range of conditions: $T_{e} \in[500-20000] \mathrm{K}$ and $T_{v}^{0} \in[100-20000] \mathrm{K}$. Three observables are calculated and discussed: population distributions, averaged vibrational energy and internal relaxation times.

\section{A. Effect of Multi-quantum transitions}

The objective of this section is the analysis of the electron-vibrational energy transfer in cooling $\left(T_{v}^{0}>T_{e}\right)$ and heating conditions $\left(T_{v}^{0}<T_{e}\right)$. To this end, the vdf of the $\mathrm{O}_{2}$ molecules at different times in the relaxation is plotted in Fig. 2 (solid blue line), for two specific test-cases. In both cases the initial distribution of the vibrational energy levels is 

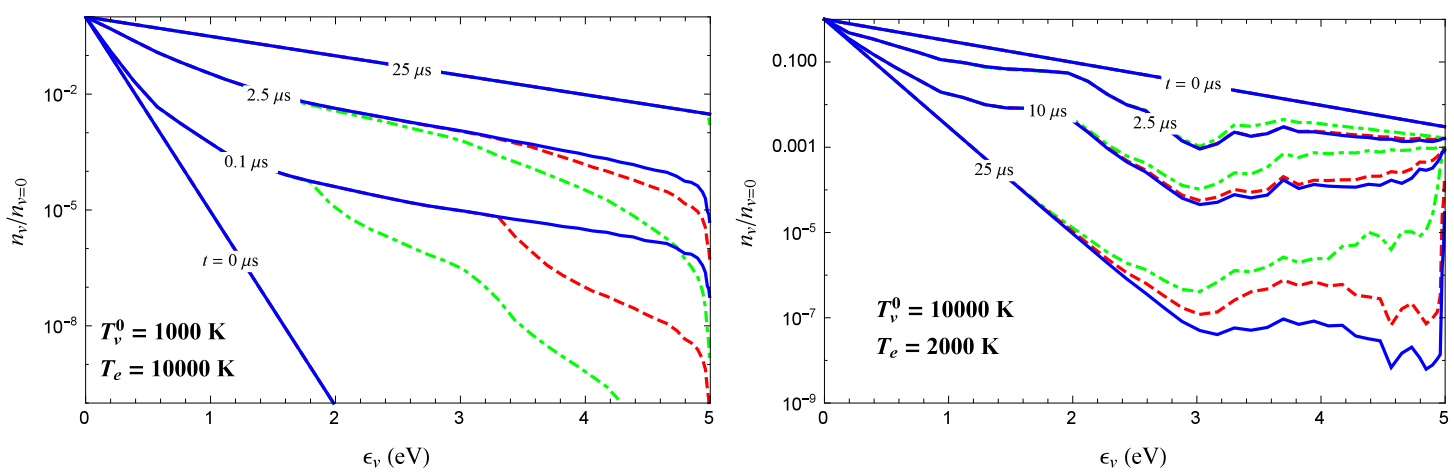

FIG. 2: Time-evolution of normalized $\mathrm{O}_{2}$ vibrational distribution function for two different cases in heating, $T_{v}^{0}<T_{e}$ (left hand side), and cooling, $T_{v}^{0}>T_{e}$ (right hand side). Solutions with full set of multi-quantum transitions allowed (solid blue line), $\Delta v=20$ (red dashed line) and $\Delta v=10$ (green dot-dashed) are shown.

assumed to be populated according to a Boltzmann distribution at temperature $T_{v}^{0}$, which is shown in Fig. 2 as a straight solid line (at $t=0 \mu \mathrm{s})$. Both cases analyzed are characterized by: a mixture number density of $n=3.210^{22}$ particles $/ \mathrm{m}^{3}$, with molar fractions of 0.995 and 0.005 for $\mathrm{O}_{2}\left(\mathrm{X}^{3} \Sigma_{g}^{-}\right)$and $e^{-}$respectively. Since dissociation is not considered, the molar fraction stays unchanged throughout the simulation.

As the time passes the distribution of the excited states is distorted, causing an overpopulation (underpopulation) of the tail in the heating (cooling) case. As the relaxation proceeds further, the population of the excited states slowly relaxes until equilibrium of the vibrational and free electron temperature is achieved. It is interesting to note that the population of the low-lying excited states is very well described by a Boltzmann distribution for most of the relaxation. This gives us confidence on the ability of reduced order model to be able to understand the energy relaxation dynamics.

In order to study the effect of multi-quantum transitions in the dynamics of reaction in (2), we have repeated the simulation including a reduced number of processes. To this end, we have introduced the parameter $\Delta v$, which limits the maximum energy transfer allowed by the inelastic collision process, i.e. such that:

$$
|v-w| \leq \Delta v,
$$

in this way only a subset of the whole set STS processes being included in the simulation. A similar approach has been developed for nitrogen in the papers in the Refs. [13, 14]. Throughout this paper, by "full" solution we indicate the results obtained with the entire set of vibrational levels, i.e. $\Delta v=42$. In Fig. 2 and 3 we show the results for the vdf and for the vibrational energy in two opposite cases of heating and cooling and we compare the behavior of the $\Delta v=20$ (red dashed line) and $\Delta v=10$ (green dot-dashed) solutions with respect to full solution (solid blue line).

In the heating case, we note a strong depletion of the high-lying vibrational levels, which are scarcely populated. This is due to a reduced efficiency of the electrons driven processes, and to their low population densities. Although the population of the high-lying vibrational levels obtained with $\Delta v=20$ and $\Delta v=10$ depart from the full solution, the time required to reach equilibrium seems to be unaffected. This can be observed in the time evolution of vibrational energy on the right side of Fig. 3, where the solutions for $\Delta v=20$ and $\Delta v=10$ 

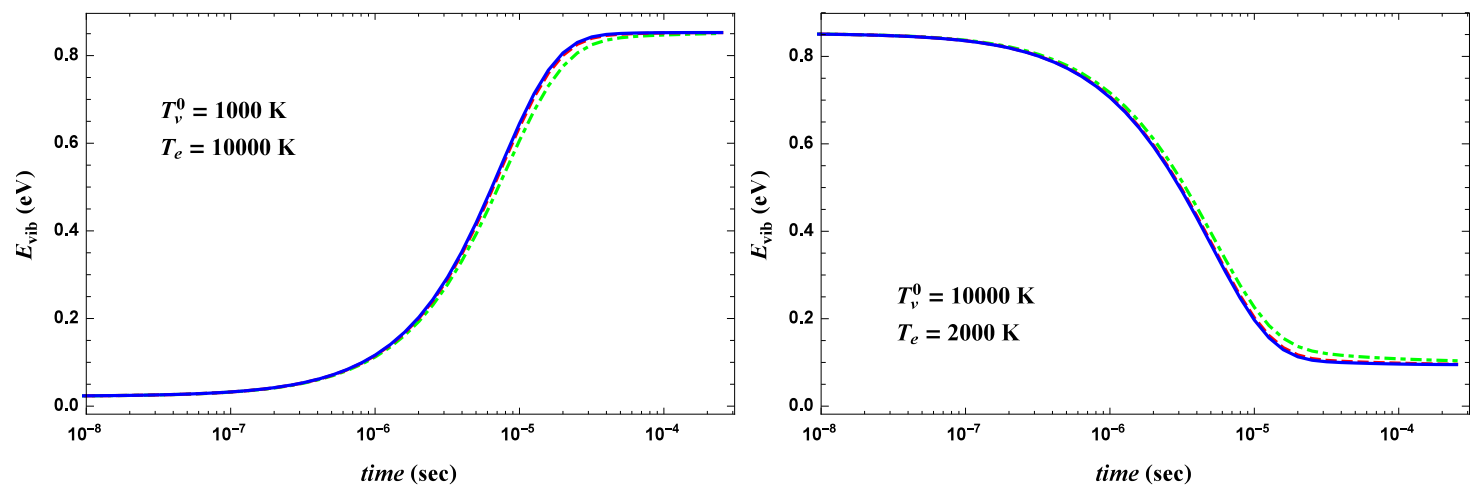

FIG. 3: Time-evolution of vibrational energy corresponding to the plots in Fig. 2.

practically follow the full solution. The consideration made in the heating case apply to the cooling case, for which the extent of non-equilibrium seems reduced. For this reason it is not further discussed.

In order to better quantify the effect of multi-quantum transitions on the relaxation process, we have calculated the differences between the full solution and the corresponding solution with different $\Delta v$ by introducing the error estimation $\varepsilon$ such that,

$$
\varepsilon=\max \frac{\left|E_{v i b}^{f u l l}(t)-E_{v i b}^{\Delta v}(t)\right|}{E_{v i b}^{f u l l}(t)},
$$

where $E_{v i b}^{f u l l}$ and $E_{v i b}^{\Delta v}$ are the solutions of Eq. (4) for the full and $\Delta v$ case starting with the same initial conditions. Plots in Fig. 4 summarize the results of calculations for the case $\Delta v=20$ and $\Delta v=10$. Two aspects can be noted: (i) As expected, in the same conditions $T_{e}$ and $T_{v}$ the error increases as the number of allowed transitions decreases, $\varepsilon(\Delta v=10)>\varepsilon(\Delta v=20)$; (ii) The error increase as the difference $\left|T_{e}-T_{v}\right|$ increase. In facts as the number of multi-quantum jumps is reduced the system needs more time to reach the equilibrium respect to the full simulation. Moreover, the maximum errors are observed in cooling, i.e. $T_{v}>T_{e}$, where the cooling of the tail of the vdf requires multi-quantum jumps. In particular the worse case is recorded for $\Delta v=10$ and $T_{v}>T_{e}$. So that in order to implement a reduced model on the vibrational level it should be used only for $\left|T_{e}-T_{v}\right| \approx 0$ and to include at least $\Delta v=20$ gap between vibrational levels.

\section{B. Relaxation time formalism}

In this section, we focus on relaxation time formalism and in particular we would like to assess the validity of the LT model, by comparing its results with ME predictions. Both approaches have been introduced in Section II.

In the ME approach, to extract the relaxation time, we solve the full STS system in Eq. (3), for fixed $T_{e}$ and $T_{v}^{0}$. From the vibrational populations of $\mathrm{O}_{2}, n_{v}(t)$, we compute the $\mathrm{O}_{2}$ averaged vibrational energy, defined by Eq. (4), and then we fit $E_{v i b}(t)$ with the analytical solution of LT equation, Eq. (5), using $\tau_{e}$ as an external parameter. In order to make the results independent of the electron density the results are provided in terms of $p_{e} \tau_{e}$. The second approach relies on the LT relaxation formula to define, $\tau_{e}$. In this case, 

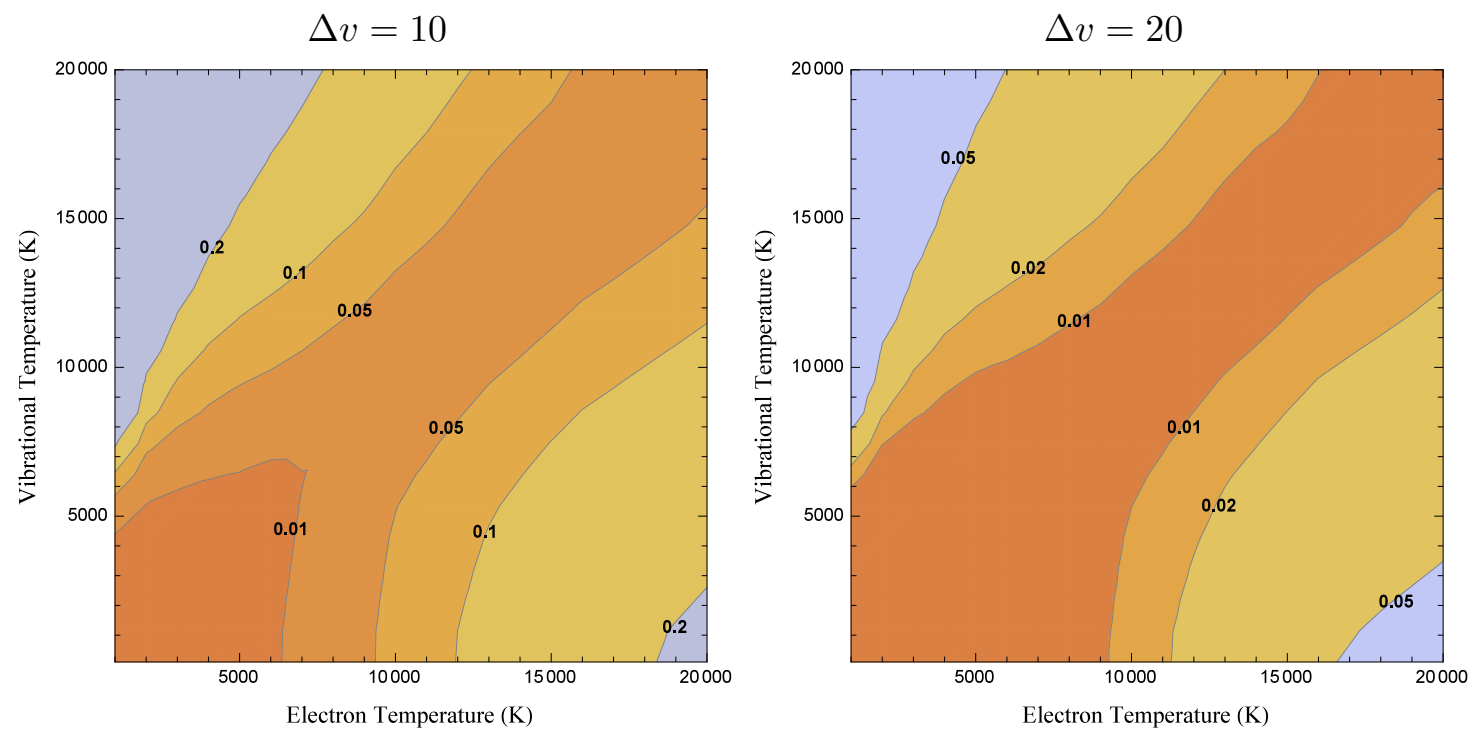

FIG. 4: Maximum error $\varepsilon$ obtained in solving time evolution in the all ranges $T_{e}$ and $T_{v}$ for $\Delta v=10$ (left hand side) and $\Delta v=20$ (right hand side).

the relaxation time can be calculated as:

$$
p_{e} \tau_{10}=\frac{k_{B} T_{e}}{k_{1,0}\left(T_{e}\right)\left(1-e^{-\theta / T_{e}}\right)},
$$

where $k_{1,0}$ is the fundamental mono-quantum rate coefficient (i.e. the $v=0$ curve in left hand side plot in Fig. 1) and $\theta=\left(\epsilon_{1}-\epsilon_{0}\right) / k_{B}=2282.01 \mathrm{~K}$ is the characteristic temperature for $\mathrm{O}_{2}$. Time relaxation, in LT formalism, depends only on electron temperature. Results for both approaches are summarized in Fig. 5.

Plot on left hand side in Fig. 5 shows a comparison of the relaxation time, for the two cases discussed above, as a function of $T_{e}$, and for different initial vibrational temperatures, $T_{v}^{0}$. At the first look, one may notice that as the relaxation time calculated from ME approach is $T_{v}^{0}$-dependent. It is interesting to observe how the LT approximation gives reasonable values of the relaxation times for the wide range of electron temperatures 2000-12000 K. Obviously the simple LT expression cannot account for the influence of the initial vibrational excitation, which appears particularly important at high $T_{e}$.

In order to better visualize the results of relaxation time, in plot on the right-hand side of Fig. 5, the time evolution of the normalized vibrational energy obtained from ME and LT approaches have been compared for a specific test-case at fixed $T_{e}=8000 \mathrm{~K}$ and for different $T_{v}^{0}$. Solid blue curves represent the ME solutions whereas dashed green lines refer to the LT model. The agreement between the two models is acceptable in particular for $T_{v}^{0}=5000,10000 \mathrm{~K}$ but at low and high $T_{v}^{0} \mathrm{LT}$ fails to reproduce the correct behaviour of the system. tion,

The $p_{e} \tau_{e}\left(T_{e}, T_{v}^{0}\right)$ relaxation times obtained from ME can be fitted by a polynomial func-

$$
p_{e} \tau_{e}\left(T_{e}, T_{v}^{0}\right)=\sum_{n=0}^{4} \sum_{m=0}^{2} c_{n, m} T_{e}^{n} T_{v}^{0^{m}},
$$

for $T_{e}$ in the range $[2000-20000] \mathrm{K}$ and $T_{v}^{0}$ in the interval $[0-20000] \mathrm{K}$. The coefficients 

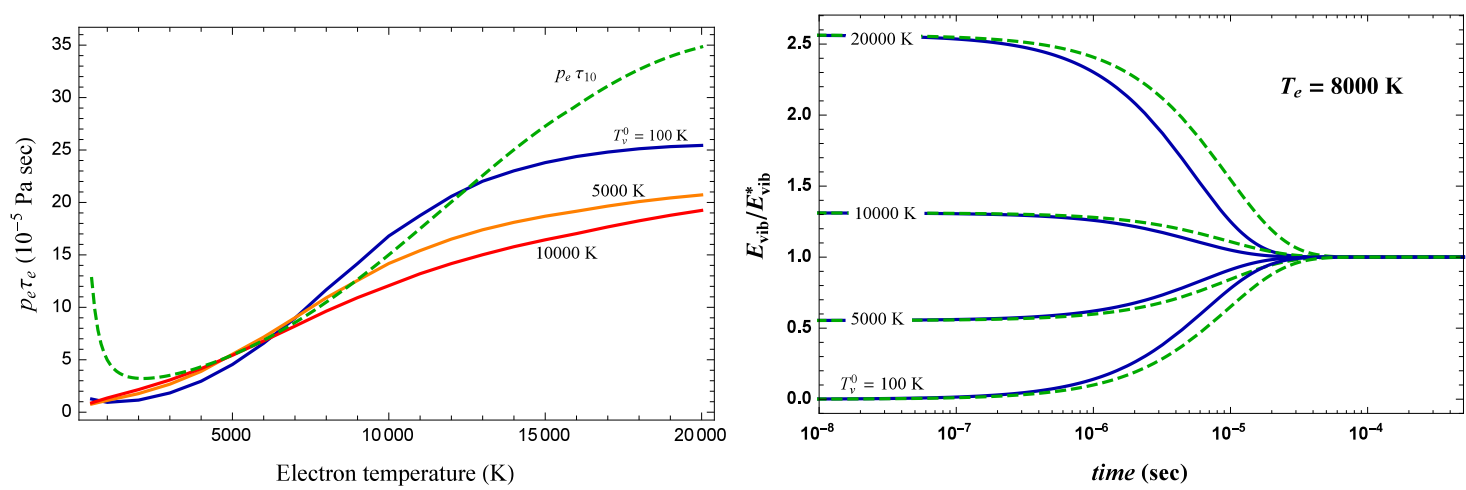

FIG. 5: On the left: Calculated $p_{e} \tau_{e}$ for different initial vibrational temperature $T_{v}^{0}$ (solid lines) compared with the Landau-Teller approximation (green dashed line). On the right: Timeevolution of normalized vibrational energy corresponding to electron temperature $T_{e}=8000 \mathrm{~K}$ for different initial vibrational temperature $T_{v}^{0}$. Solid blue lines refer to the master equation solutions, whereas dashed green lines refer to the Landau-Teller results.

\begin{tabular}{|c|rrr|}
\hline $\mathrm{m}$ & 0 & 1 & \multicolumn{1}{c|}{2} \\
\hline 0 & 2.418 & $-2.66010^{-4}$ & $1.01510^{-8}$ \\
1 & $-2.09410^{-3}$ & $3.53710^{-7}$ & $-1.15310^{-11}$ \\
2 & $7.17410^{-7}$ & $-7.57810^{-11}$ & $2.42210^{-15}$ \\
3 & $-4.51110^{-11}$ & $4.43510^{-15}$ & $-1.40710^{-19}$ \\
4 & $8.68210^{-16}$ & $-8.13110^{-20}$ & $2.61010^{-24}$ \\
\hline
\end{tabular}

TABLE II: Coefficients $c_{n, m}$ for the two-dimensional fit for $p_{e} \tau_{e}$ in Eq. (10).

$c_{n, m}$ are given in Table II.

Finally, in Fig. 6 we show the comparison between the present results for $p_{e} \tau_{e}$ for oxygen and the corresponding results for nitrogen taken form Ref. [14]. From the figure it is easy to see how the vibrational relaxation of nitrogen molecules is significantly faster than the oxygen vibrational relaxation for temperature exceeding 7000-8000 K. This suggests the possible existence of non-equilibrium between the vibrational energies of molecular oxygen and nitrogen molecules, interacting with electron gas for condition of interest to hypersonic entry applications. However, it is important to notice that, to validity of this conclusion is limited only to the electron driven processes, considered in this analysis. Only a more comprehensive analysis including both electron and heavy particle driven processes will be able to shed some light on the existence of vibrational non-equilibrium between the vibrational energies of nitrogen and oxygen.

\section{CONCLUSIONS}

We have performed extensive non-empirical calculations for oxygen energy transfer, which simulate an experiment conducted in an idealized isothermal chemical reactor, with free electrons as a collision partner. We solved the master equation for the entire vibrational structure of $\mathrm{O}_{2}\left(\mathrm{X}^{3} \Sigma_{g}^{-}\right)$molecules allowing us to accurately describe the internal energy re- 


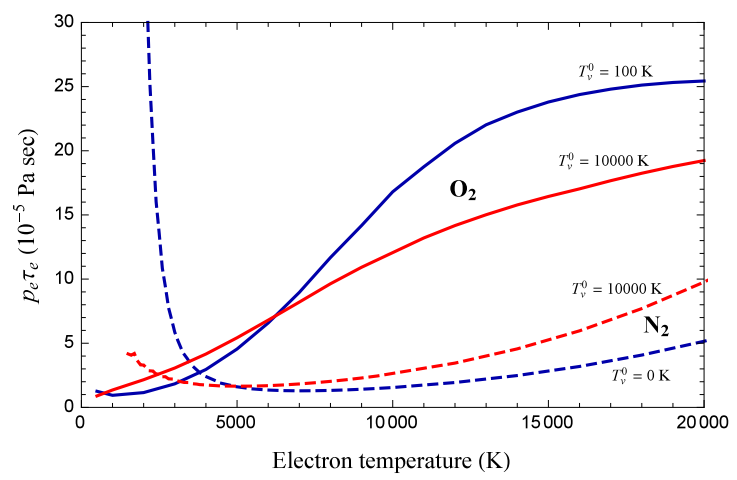

FIG. 6: Comparison between calculated $p_{e} \tau_{e}$, as a function of the electron temperature, for oxygen (solid lines) and the corresponding results for nitrogen (dashed lines) [14] for two different initial vibrational temperatures.

laxation processes for a wide rage of temperatures $(0$ - $20000 \mathrm{~K})$. The state-to-state approach adopted in this work relies on the knowledge of the vibrational state-to-state transfer rate coefficients, computed as part of the ab-initio database developed at the University of Bari and part of the Phys4Entry project.

The modeling of the State-to-state processes in the chemical reactor has shown that the distribution of the vibrationally excited states strongly deviates from the equilibrium vibrational distributions, during the relaxation both in heating and cooling conditions. Although not considered here, this can have a strong influence on the chemical kinetics of reactive plasma flows, in particular where vibrationally favored reactions (e.g., dissociation) play a role.

We have discussed the possibility to model the relaxation by a linear rate equation, the Landau-Teller formula, with a single relaxation time. The results obtained from the relaxation formalism and from the full kinetic model are compared to conclude that the former can be used with sufficient accuracy to model the vibrational energy relaxation in most cases of practical interest. Analytical fits of the relaxation time, that depends both on free electron and vibrational temperatures, has been provided.

Finally, we compared the characteristic relaxation time of $e-\mathrm{N}_{2}\left(\mathrm{X}^{1} \Sigma_{\mathrm{g}}^{+}\right)$and $e-\mathrm{O}_{2}\left(\mathrm{X}^{3} \Sigma_{g}^{-}\right)$ systems. We have shown that molecular nitrogen molecules relax at faster rates, especially for high temperatures.

\section{Acknowledgments}

The authors have benefited from numerous discussions with Dr. D. Bruno (Istituto di Nanotecnologia, CNR, Bari, Italy). V.L. wish to thank Prof. R. Celiberto (Politecnico di Bari, Italy) for careful reading of the paper and Dr. E. Josyula (Air Force Research Laboratory, Dayton, Ohio) for useful discussions. Research of V.L. was funded by Ohio Aerospace Institute, grant agreement project \#WE202270. The research of M.P. is based in part upon work supported by the Department of Energy, National Nuclear Security Administration, under Award Number DE-NA0002374. 


\section{References}

[1] P.A. Gnoffo. Annual Review of Fluid Mechanics, 31(1):459-494, 1999.

[2] S.T. Surzhikov. Journal of Heat Transfer, 134(3):031002-031002, January 2012.

[3] A.Bultel, B.G. Cheron, A. Bourdon, O. Motapon, and I.F. Schneider. Physics of Plasmas, 13 (4):043502, 2006.

[4] A. Munafò, A. Lani, A. Bultel, and M. Panesi. Physics of Plasmas, 20(7), 2013.

[5] I.V. Adamovich and W.R. Lempert. Plasma Physics and Controlled Fusion, 57(1):014001, 2015.

[6] K.M. Aggarwal, N. Nath, and C.S.G.K. Setty. Planetary and Space Science, 27(6):753 - 768, 1979.

[7] S.S. Prasad and D.R. Furman. Journal of Geophysical Research, 78(28):6701-6707, 1973.

[8] P. Stubbe and W.S. Varnum. Planetary and Space Science, 20(8):1121 - 1126, 1972.

[9] N.F. Lane and A. Dalgarno. Journal of Geophysical Research, 74(11):3011-3012, 1969.

[10] D.B. Jones, L. Campbell, M.J. Bottema, and M.J. Brunger. New Journal of Physics, 5(1): 114, 2003.

[11] M. Panesi, R.L. Jaffe, D. Schwenke, and T.E. Magin. J. Chem. Phys., 138:044312, 2013.

[12] A. Munafò, M. Panesi, R. L. Jaffe, G. Colonna, A. Bourdon, and T.E. Magin. Eur. Phys. Jour. D, 66:188, 2012.

[13] Mario Lino Da Silva, Vasco Guerra, and Jorge Loureiro. Journal of Thermophysics and Heat Transfer, 21(2):303-310, April 2007.

[14] V. Laporta and D. Bruno. The Journal of Chemical Physics, 138(10):104319, 2013.

[15] K.L. Heritier, R.L. Jaffe, V. Laporta, and M. Panesi. The Journal of Chemical Physics, 141 (18):-, 2014.

[16] M. Capitelli, G. Colonna, G. D'Ammando, V. Laporta, and A. Laricchiuta. Physics of Plasmas, 20(10):-, 2013.

[17] M.W. Huo. Non-equilibrium Processes in Partially Ionized Gases, Plenum, New York, 1990. 341-348 pp.

[18] J.H. Lee. Journal of Thermophysics and Heat Transfer, 7(3):399-405, 1993.

[19] J.D. Mertens. Journal of Thermophysics and Heat Transfer, 13(2):204-209, APR-JUN 1999.

[20] A. Bourdon and P. Vervisch. Phys. Rev. E, 55(4):4634, 1997.

[21] V. Laporta, R. Celiberto, and J.M. Wadehra. Plasma Sources Science and Technology, 21(5): 055018, 2012.

[22] V. Laporta, D.A. Little, R. Celiberto, and J. Tennyson. Plasma Sources Science and Technology, 23(6):065002, 2014.

[23] M. Capitelli, I. Armenise, E. Bisceglie, D. Bruno, R. Celiberto, G. Colonna, G. D'Ammando, O. De Pascale, F. Esposito, C. Gorse, V. Laporta, and A. Laricchiuta. Plasma Chemistry and Plasma Processing, 32:427-450, 2012. 10.1007/s11090-011-9339-7.

[24] G.J. Schulz. Phys. Rev. E, 125:229-232, 1963.

[25] D.G. Thompson. Adv. At. Mol. Phys., 19:309, 1983.

[26] L.A. Morgan. J. Phys.B: At. Mol. Phys., 19:439-445, 1986.

[27] V. Laporta, R. Celiberto, and J. Tennyson. Plasma Sources Science and Technology, 22(2): 025001, 2013. 
[28] V. Laporta, R. Celiberto, and J. Tennyson. Phys. Rev. A, 91:012701, Jan 2015.

[29] M. Allan. Journal of Physics B: Atomic, Molecular and Optical Physics, 28(23):5163, 1995.

[30] Database of the european union phys4entry project, 2012-2015.

http://users.ba.cnr.it/imip/cscpal38/phys4entry/database.html.

[31] L.D. Landau and E. Teller, Phys. Z. Sowjetunion 10, 3440 (1963). 